\title{
Almost Sure Exponential Stabilization of Stochastic Systems by State-Feedback Control ${ }^{\star}$
}

\author{
Liangjian $\mathrm{Hu}^{\mathrm{a}, \mathrm{b}}$, Xuerong $\mathrm{Mao}{ }^{\mathrm{b}}$ \\ ${ }^{a}$ Department of Applied Mathematics, Donghua University, Shanghai 200051, China \\ ${ }^{\mathrm{b}}$ Department of Statistics and Modelling Science, University of Strathclyde, Glasgow G1 1XH,UK
}

\begin{abstract}
So far, a major part of the literature on the stabilisation issues of stochastic systems has been dedicated to mean square stability. This paper develops a new class of criteria for designing a controller to stabilise a stochastic system almost surely which is unable to be stabilised in mean-square sense. The results are expressed in terms of linear matrix inequalities (LMIs) which are easy to be checked in practice by using MATLAB Toolbox. Moreover, the control structure in this paper appears not only in the drift part but also in the diffusion part of the underlying stochastic system.
\end{abstract}

Key words: stochastic stability; stabilisation; mean square stability; almost sure stability; ; linear matrix inequality (LMI).

\section{Introduction}

As it is well-known, there are several different concepts of stability in the literature on stochastic systems, such as asymptotic stability in probability, almost sure exponential stability and mean square exponential stability etc. Generally speaking, mean square exponential stability and almost sure exponential stability do not imply each other, although both of them imply asymptotic stability in probability. However, in many situations, e.g. linear systems, mean square exponential stability implies almost sure exponential stability (Mao, 1997).

In the last decades, a major part of the literature on the stabilisation issues of stochastic systems has been dedicated explicitly or implicitly to mean square stability (El Ghaoui, 1995; Wang, Qiao \& Burnham, 2002; Xu \& Chen, 2002; Chen \& Zhang, 2004; Dragan, Morozan \& Stoica, 2004; Yue \& Han, 2005; Xu etal, 2006). Upon the mean square stability concept, noises always pay a destabilisation impact on system stability, namely, an unstable system is noway stabilised by bringing in noises. On the other hand, the literature on the stability and stabilisation issues of mechanics system (Roberts

* This paper was not presented at any IFAC meeting. Corresponding author L. J. Hu. Tel. +86-21-67792089. Fax +8621-67792085.

Email addresses: Ljhu@dhu.edu.cn (Liangjian $\mathrm{Hu}$ ), xuerong@stams.strath.ac.uk (Xuerong Mao).
\& Spanos, 1986; Nolan \& Sri Namachchivaya, 1999; Tylikowski, 2005), jump parameter systems (Ezzine \& Kavranoglyu, 1997; Fang, 1997; Fang \& Loparo, 2002; Lee \& Dullerudb, 2006) and finance market systems (Fernholz \& Karatzas, 2005) has been frequently addressed on almost sure stability.

Since 1980's, it has been observed that noise can be used to stabilise a given unstable system almost surely (Khasminski, 1980). The research implies that noise can also play a stabilisation role for system stability. Systems which are unable or very difficult to be mean square stabilised may be stabilised almost surely by utilising noise signal.

For instance, take into account a simple one-dimensional linear stochastic control system

$$
d x(t)=(a x(t)+b u(t)) d t+(c x(t)+f u(t)) d w(t),
$$

where $a, b, c, f$ are scalar constants. Consider a statefeedback control $u(t)=k x(t)$, the explicit solution of the closed-loop system

$$
d x(t)=(a+b k) x(t) d t+(c+f k) d w(t)
$$

with initial data $x(0)=x_{0}$ is

$$
x(t)=x_{0} \exp \left\{\left[a+b k-(c+f k)^{2} / 2\right] t+(c+f k) w(t)\right\},
$$


whose second moment is

$$
\mathcal{E}[x(t)]^{2}=x_{0}^{2} \exp \left\{\left[2(a+b k)+(c+f k)^{2}\right] t\right\} .
$$

It is evident that the second moment will tend to infinite if $a+b k>0$ when the deterministic part is not stable, nonetheless, the sample paths tend to the origin almost surely if $a+b k-(c+f k)^{2} / 2<0$. An interesting case is $b=0$ when the deterministic part is not stabilizable and the stochastic system is not mean square stabilizable, while there are many choices of a feedback gain $k$ to stabilise the system path-wisely, that is to say, the system can be stabilised by noise almost surely or with probability one.

The problems of stabilisation of differential equations by noise have been studied by many authors and we here mention Arnold, Crauel \& Wihstutz (1983); Pardoux \& Wihstutz (1992); Mao (1994); Kwiecińska (2002); Appleby \& Mao (2005); Caraballo \& Robinson (2004); Yuan $\&$ Mao (2004). The results demonstrate that after adding a stochastic term to a deterministic differential equation the top Lyapunov exponent becomes smaller, i.e. the stochastic system turns to be more stable than the deterministic one. However, these studies have not taken the structure of feedback control (which is in terms of control engineering) into account. There are a few recent papers dealing with the control design problem in the almost sure sense, for example, Yuan \& Lygeros (2005) but the criteria there are in terms of nonlinear matrix inequalities.

In this paper, we will develop a class of LMI conditions for designing a controller to stabilise a stochastic system almost surely which may not able to be stabilised in mean-square sense. The LMI conditions are easy to check in practice by using MATLAB Toolbox. Moreover, the control structure of this paper appears not only in shift part as in Yuan \& Mao (2004) and Yuan \& Lygeros (2005) but also in diffusion part of the stochastic system. We will comment a bit more about this issue in Section 2 below.

The rest of the paper is organised as follows. Some definitions and lemmas on stochastic stability are recalled in Section 2. In Section 3, we investigate the almost sure stabilisation problem of stochastic linear time invariant (SLTI) systems, where the design method is deduced to LMIs. An example is discussed for illustration in Section 4. Finally, we conclude the paper in Section 5.

\section{The Concepts of Stochastic Stability}

Notations: Throughout this paper, $\mathbb{R}^{n}$ and $\mathbb{R}^{m \times n}$ denote, respectively, the $n$ dimensional Euclidean space and the set of $m \times n$ real matrices. $|\cdot|$ denotes the Euclidean norm in $\mathbb{R}^{n}$. $I_{n}$ denotes the identity matrix of dimension $n$ (Sometimes, the subscript $n$ is omitted when no confusion can arise). $A^{T}$ denotes the transpose of vector or matrix $A$ and $\|A\|$ is the operator norm of $A$, i.e. $\|A\|=\sup _{|x|=1}\{A x\}=\sqrt{\lambda_{\max }\left(A^{T} A\right)}$. For a symmetric matrix $A$ in $\mathbb{R}^{n \times n}, \lambda_{\min }(A)$ and $\lambda_{\max }(A)$ mean the smallest and largest eigenvalue, respectively. For symmetric matrices $P$ and $Q, P>0$ means that $P$ is positive definite, $P>Q$ means $P-Q>0$. Symbols $\geq,<$, and $\leq$ for matrices are defined similarly. Matrices, if not explicitly stated, are assumed to have compatible dimensions. A star symbol ${ }^{*}$ ' in a symmetric matrix denotes the transposed element at the symmetric position.

Consider the following stochastic differential equation

$$
d x(t)=f(x(t)) d t+g(x(t)) d w(t), \quad t \geq 0,
$$

with $x(0)=x_{0} \in \mathbb{R}^{n}$, where $x(t) \in \mathbb{R}^{n}$ denotes the state vector, and $w(t)=\left[w_{1}(t), w_{2}(t), \cdots, w_{m}(t)\right]^{T}$ denotes an $m$-dimensional Brownian motion or Wiener process. The vector or matrix-valued functions $f(x)$ and $g(x)$ are assumed to be of appropriate dimensions. The solution of (5) is denoted by $x\left(t, x_{0}\right)$ or $x(t)$. For the purpose of stability study, we assume that $f(0)=0$ and $g(0)=$ 0 . Hence the origin $x(t) \equiv 0$ is the trivial solution or equilibrium of (5).

Definition 1. 1) The equilibrium of (5) is said to be mean square exponentially stable (m.s. stable, for short) if there exists a pair of positive constants $\lambda$ and $\alpha$ such that

$$
\mathcal{E}\left|x\left(t, x_{0}\right)\right|^{2} \leq \lambda\left|x_{0}\right|^{2} \exp (-\alpha t)
$$

for all $t \geq 0$ and $x_{0} \in \mathbb{R}^{n}$. Namely,

$$
\limsup _{t \rightarrow \infty} \frac{1}{t} \log \mathcal{E}\left|x\left(t, x_{0}\right)\right|^{2}<0
$$

for all $x_{0} \in \mathbb{R}^{n}$.

2) The equilibrium of (5) is said to be almost surely exponentially stable (a.s. stable, for short) if

$$
\mathcal{P}\left(\limsup _{t \rightarrow \infty} \frac{1}{t} \log \left|x\left(t, x_{0}\right)\right|<0\right)=1
$$

for all $x_{0} \in \mathbb{R}^{n}$.

The mean square exponential stability can ensure the internal stability defined in Hinrichsen \& Pritchard (1998). Both the mean square exponential stability and the almost sure exponential stability imply the globally asymptotic stability(GAS) in Deng \& Krstic (1997), and Pan \& Basar (1999). Under some usual conditions (Mao, 1997), the mean square exponential stability implies the almost sure exponential stability.

Let $\mathcal{C}^{2}\left(\mathbb{R}^{n}\right)$ denote the family of all scalar function $V(x)$ defined on $\mathbb{R}^{n}$ which are twice continuously differentiable 
in $x$. Two Itô stochastic differential operators on $\mathcal{C}^{2}\left(\mathbb{R}^{n}\right)$ are defined as

$$
\begin{aligned}
\mathcal{L} V(x) & =\frac{\partial V}{\partial x}(x) f(x) \\
& +\frac{1}{2} \operatorname{trace}\left[g^{T}(x) \frac{\partial^{2} V}{\partial x^{2}}(x) g(x)\right], \\
\mathcal{H} V(x) & =\frac{\partial V}{\partial x}(x) g(x) .
\end{aligned}
$$

According to the Itô stochastic differential rule, we have

$$
d V(x(t))=\mathcal{L} V(x(t)) d t+\mathcal{H} V(x(t)) d w(t) .
$$

Lemma 1. (Mao, 1997)[page 121] Assume that there exists a function $V \in \mathcal{C}^{2}\left(\mathbb{R}^{n}\right)$ and constants $p>0, c_{1}>$ $0, c_{2} \in \mathbb{R}, c_{3} \geq 0$ such that

$$
\begin{aligned}
& V(x) \geq c_{1}|x|^{p} \\
& \mathcal{L} V(x) \leq c_{2} V(x), \\
& (\mathcal{H} V(x))^{2} \geq c_{3} V^{2}(x), \\
& c_{3}>2 c_{2}
\end{aligned}
$$

for all $x \in \mathbb{R}^{n}$. Then the equilibrium of (5) is almost surely exponentially stable.

Note that $\mathcal{L} V(x)$ in Lemma 1 could be positive. This surprising attribute differentiates the almost sure exponential stability from both exponential stability of deterministic system and mean-square exponential stability of stochastic system.

\section{Almost Sure Stabilisation}

Given an unstable stochastic linear time invariant (SLTI) system

$$
d x(t)=A x(t) d t+\sum_{i=1}^{m} C_{i} x(t) d w_{i}(t)
$$

we are required to define a state-feedback control $u(t)$ so that the corresponding controlled system

$d x(t)=[A x(t)+B u(t)] d t+\sum_{i=1}^{m}\left[C_{i} x(t)+D_{i} u(t)\right] d w_{i}(t)$

become a.s. stable. Here $A$ and $C_{i}$ 's are in $\mathbb{R}^{n \times n}$ while $B$ and $D_{i}$ 's in $\mathbb{R}^{n \times l}$ and the control $u(t)$ is $\mathbb{R}^{l}$-valued. We note that the control $u(t)$ appears in both shift and diffusion parts, although in many papers it appears only in the shift part (see e.g. Yuan \& Mao (2004); Yuan \& Lygeros (2005)) or in the diffusion part (see e.g. Arnold, Crauel \& Wihstutz (1983); Pardoux \& Wihstutz (1992); Mao (1994)). The reader may wonder if this is a simplification of the problem, since the more control power you have the easier it is to achieve the a.s. stabilising controller. But there are two reasons for us to do so: (i) There are lots of systems which cannot be stabilised almost surely if the control is restricted only in shift or diffusion part (see Example 1 in Section 4). (ii) The theory developed in this paper can be applied directly to the case when the control is only in shift or diffusion part (see Corollary 1 in Section 3).

In this paper, we look for a linear state-feedback control of the form $u(t)=K x(t)$, where $K \in \mathbb{R}^{l \times n}$. Hence, the closed-loop system is

$$
d x(t)=(A+B K) x(t) d t+\sum_{i=1}^{m}\left(C_{i}+D_{i} K\right) x(t) d w_{i}(t) .
$$

The stabilisation problem is therefore to find a matrix $K$ for the closed-loop system to be a.s. stable.

Many known results are concerned with the mean-square exponential stabilisation of the system. For example, we cite the following results from El Ghaoui (1995).

Theorem 1. (El Ghaoui, 1995) The equilibrium of the stochastic system (12) is mean-square exponentially stable with respect to state-feedback gain $K=Y X^{-1}$, if there exists a positive definite matrix $X$ and a matrix $Y$ such that the following LMI holds

$$
\left(\begin{array}{cc}
\Pi_{11} & * \\
\Pi_{21} & \Pi_{22}
\end{array}\right)<0
$$

where $\Pi_{11}=(A X+B Y)^{T}+(A X+B Y), \Pi_{21}=\left[\left(C_{1} X+\right.\right.$ $\left.\left.D_{1} Y\right)^{T},\left(C_{2} X+D_{2} Y\right)^{T}, \cdots,\left(C_{m} X+D_{m} Y\right)^{T}\right]^{T}, \Pi_{22}=$ $\operatorname{diag}(-X,-X, \cdots,-X)$.

Of course, under the condition of Theorem 1, the equilibrium of system (12) is also almost surely exponentially stable. However, the underlying system may not be stabilised in the mean-square sense. In this case, our following new results may be used to design a statefeedback controller to stabilise the systems in the almost sure sense.

Theorem 2. The equilibrium of the stochastic system (12) is almost surely exponentially stable with respect to state-feedback gain $K=Y X^{-1}$, if there exists a positive definite matrix $X$, a matrix $Y$ and real number $\alpha_{i} \geq 0$ $(1 \leq i \leq m)$ such that following LMIs hold:

$$
\left(\begin{array}{cc}
\Pi_{11}-\alpha X & * \\
\Pi_{21} & \Pi_{22}
\end{array}\right)<0
$$

and, for each $i=1,2, \cdots, m$, either

$\left(C_{i} X+D_{i} Y\right)^{T}+\left(C_{i} X+D_{i} Y\right)-\sqrt{2 \alpha_{i}} X>0$

or

$$
\left(C_{i} X+D_{i} Y\right)^{T}+\left(C_{i} X+D_{i} Y\right)+\sqrt{2 \alpha_{i}} X<0,
$$


where $\Pi_{11}, \Pi_{21}, \Pi_{22}$ are the same as those in Theorem 1 and $\alpha=\sum_{i=1}^{m} \alpha_{i}$.

Proof. Set $P=X^{-1}>0$ and define $V(x)=x^{T} P x$ for $x \in \mathbb{R}^{n}$. Clearly, equation (9a) is satisfied with $p=2$ and $c_{1}=\lambda_{\min }(P)$. Applying operator (6), we compute

$$
\begin{aligned}
\mathcal{L} V(x) & =\frac{\partial V}{\partial x}(A+B K) x \\
& +\frac{1}{2} \sum_{i=1}^{m}\left[\left(C_{i}+D_{i} K\right) x\right]^{T} \frac{\partial^{2} V}{\partial x^{2}}\left(C_{i}+D_{i} K\right) x \\
& =x^{T}\left[(A+B K)^{T} P+P(A+B K)\right. \\
& \left.+\sum_{i=1}^{m}\left(C_{i}+D_{i} K\right)^{T} P\left(C_{i}+D_{i} K\right)\right] x .
\end{aligned}
$$

Thus, there exists a $c_{2}<\alpha$ such that equation (9b) will hold if

$$
\begin{aligned}
& (A+B K)^{T} P+P(A+B K) \\
+ & \sum_{i=1}^{m}\left(C_{i}+D_{i} K\right)^{T} P\left(C_{i}+D_{i} K\right)-\alpha P<0 .
\end{aligned}
$$

Noting that $X=P^{-1}$ and $Y=K X$, and pre- and postmultiplying (15) by $X$ yields

$$
\begin{aligned}
& (A X+B Y)^{T}+A X+B Y \\
+ & \sum_{i=1}^{m}\left(C_{i} X+D_{i} Y\right)^{T} X^{-1}\left(C_{i} X+D_{i} Y\right)-\alpha X<0,
\end{aligned}
$$

which is equivalent to (14a) by the Schur complement lemma (Boyd etal, 1994). In other words, (9b) with $c_{2}<$ $\alpha$ is guaranteed by (14a). To verify ((9c), we compute, by equation ( 7$)$, that

$$
\mathcal{H} V(x)=2 x^{T} P\left(\left(C_{1}+D_{1} K\right) x, \cdots,\left(C_{m}+D_{m} K\right) x\right),
$$

which gives

$(\mathcal{H} V(x))^{2}=\sum_{i=1}^{m}\left[x^{T}\left(\left(C_{i}+D_{i} K\right)^{T} P+P\left(C_{i}+D_{i} K\right)\right) x\right]^{2}$.

Hence (9c) will hold with $c_{3}=2 \alpha$ if, for each $i=$ $1,2, \cdots, m$, either

$$
\left(C_{i}+D_{i} K\right)^{T} P+P\left(C_{i}+D_{i} K\right)-\sqrt{2 \alpha_{i}} P>0
$$

or

$$
\left(C_{i}+D_{i} K\right)^{T} P+P\left(C_{i}+D_{i} K\right)+\sqrt{2 \alpha_{i}} P<0 .
$$

But these are equivalent to (14b) or (14c), respectively. In other words, $(9 \mathrm{c})$ with $c_{3}=2 \alpha$ is guaranteed by $(14 \mathrm{~b})$ or $(14 \mathrm{c})$. Finally, we clearly have $c_{3}>2 c_{2}$. We have therefore verified all the conditions of Lemma 1 so the assertion of this theorem follows.

Let us now return to the case where the control is restricted only in shift or diffusion part. For illustration we only consider the later, namely the stochastic controlled system

$$
d x(t)=A x(t) d t+\sum_{i=1}^{m}\left(C_{i}+D_{i} K\right) x(t) d w_{i}(t)
$$

For this we have the following corollary which follows from Theorem 2 simply by setting $B=0$.

Corollary 1. The equilibrium of the controlled system (16) is almost surely exponentially stable with respect to state-feedback gain $K=Y X^{-1}$, if there exists a positive definite matrix $X$, a matrix $Y$ and real number $\alpha_{i} \geq 0$ $(1 \leq i \leq m)$ such that the LMIs (14a) and either (14b) or (14c) hold but $\Pi_{11}$ in (14a) is now defined by $\Pi_{11}=$ $(A X)^{T}+A X$.

Before we proceed to study more general problem, let us discuss an example to illustrate our theory so far.

Example 1. Consider a 2-dimensional linear system

$$
d x(t)=A x(t) d t+C_{1} x(t) d w_{1}(t)
$$

where

$$
A=\left(\begin{array}{ll}
3 & 1 \\
0 & 1
\end{array}\right), \quad C_{1}=\left(\begin{array}{cc}
3 & 0.2 \\
0 & 1
\end{array}\right) .
$$

It is easy to show that this system is a.s. unstable (Fig.1). Assume that we are required to define a state-feedback control $u(t)=B K x(t)$ only in the shift part so that the corresponding controlled system

$$
d x(t)=[A+B K) x(t) d t+C_{i} x(t) d w_{1}(t)
$$

becomes a.s. stable, where $B=(1,0)^{T}$ is given while $K \in \mathbb{R}^{1 \times 2}$ is to be designed. However, given the structure of $B$, it is easy to see there is no such $K$ for equation (18) to be a.s. stable, since $x_{2}(t)$ obeys

$$
d x_{2}(t)=x_{2}(t) d t+x_{2}(t) d w_{1}(t)
$$

whence $x_{2}(t) \rightarrow \infty$ a.s.

Let us now further consider the stochastic controlled system

$$
d x(t)=(A+B K) x(t) d t+\left(C_{i}+D_{1} K\right) x(t) d w_{1}(t),
$$

where $D_{1}=(-1,2)^{T}$. Noting that $d \mathcal{E} x_{2}(t) / d t=\mathcal{E} x_{2}(t)$, we observe that $\mathcal{E} x_{2}(t) \rightarrow \infty$ as $t \rightarrow \infty$ for any $x_{2}(0) \neq 0$ and any $K$. This means that the system is unstabilizable 

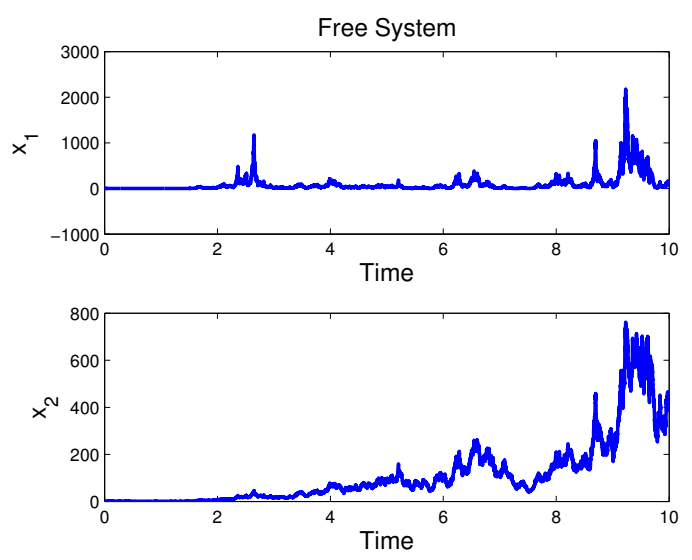

Fig. 1. Unstable System
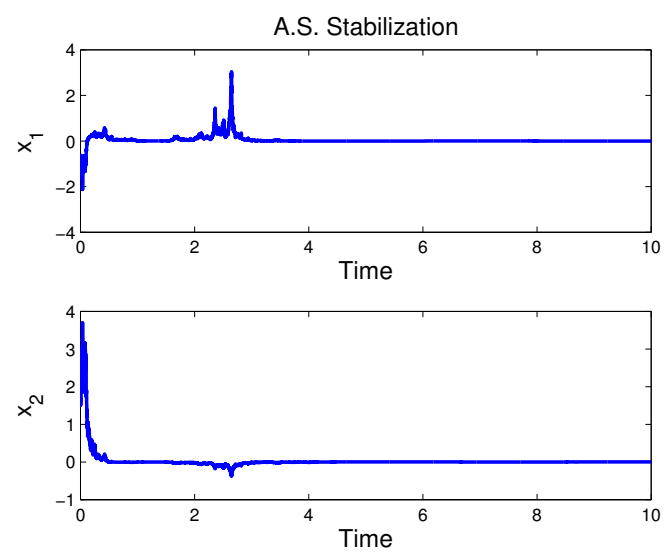

Fig. 2. a.s. Stabilisation

in mean-square sense. However, by setting $h=1$, and solving LMIs (14a) and (14b) for $\alpha=h, 2 h, \cdots$, we find

$$
\alpha=15, \quad X=\left(\begin{array}{cc}
0.3786 & -0.0153 \\
-0.0153 & 0.1770
\end{array}\right)
$$

and $Y=(0.0290,0.1886)$. By Theorem 2 we can conclude that chosing

$$
K=(0.1203,1.0760)
$$

we make system (19) a.s. stable (Fig.2).

\section{Stabilization of Uncertain Systems}

Let us now generalise our theory to cope with the uncertainty of system parameters. More precisely, let us con- sider the uncertain stochastic control system of the form

$$
\begin{aligned}
d x(t) & =[(A+\Delta A) x(t)+(B+\Delta B) u(t)] d t \\
& +\sum_{i=1}^{m}\left[\left(C_{i}+\Delta C_{i}\right) x(t)\right. \\
& \left.+\left(D_{i}+\Delta D_{i}\right) u(t)\right] d w_{i}(t),
\end{aligned}
$$

with the state-feedback control $u(t)=K x(t)$. The closed-loop system is

$$
\begin{aligned}
d x(t) & =(A+\Delta A+(B+\Delta B) K) x(t) d t \\
& +\sum_{i=1}^{m}\left(C_{i}+\Delta C_{i}+\left(D_{i}+\Delta D_{i}\right) K\right) x(t) d w_{i}(t) .
\end{aligned}
$$

Here $A, B$ etc. are the same as before while the uncertain matrices are assumed to have the following structures

$$
\begin{gathered}
\Delta A=N_{0} F_{0} N_{A}, \quad \Delta B=N_{0} F_{0} N_{B}, \\
\Delta C_{i}=N_{i} F_{i} N_{C_{i}}, \quad \Delta D_{i}=N_{i} F_{i} N_{D_{i}}
\end{gathered}
$$

for $i=1,2, \cdots, m$, where $N_{0}, N_{A}, N_{B}, N_{i}, N_{C_{i}}, N_{D_{i}}$ are known real constant matrices with appropriate dimensions but $F_{i}$ 's are unknown and obey $F_{i}^{T} F_{i} \leq I$. Such structure uncertainty has been used by many authors e.g. Wang, Qiao \& Burnham (2002); Xu \& Chen (2002).

The following theorem provides an LMI method to design a robust controller to stabilize the uncertain system almost surely.

Theorem 3. The equilibrium of the uncertain stochastic system (21) is almost surely exponentially stable with respect to state-feedback gain $K=Y X^{-1}$, if there exists a positive definite matrix $X$, a matrix $Y$ and positive scalars $\alpha_{i}, \gamma, \varepsilon_{i}, \delta_{i}(i=1,2, \cdots, m)$ such that the following LMIs hold:

$$
\left(\begin{array}{cccc}
\Pi_{11}-\alpha X & * & * & * \\
N_{A} X+N_{B} Y & -\gamma I & * & * \\
\Pi_{31} & 0 & \Pi_{33} & * \\
\Pi_{41} & 0 & 0 & \Pi_{44}
\end{array}\right)<0
$$

and, for each $i=1,2, \cdots, m$, either

$$
\left(\begin{array}{cc}
\Omega_{i}-\delta_{i} N_{i} N_{i}^{T}-\sqrt{2 \alpha_{i}} X & * \\
\left(N_{C_{i}} X+N_{D_{i}} Y\right)^{T} & \delta_{i} I
\end{array}\right)>0,
$$

or

$$
\left(\begin{array}{cc}
\Omega_{i}+\delta_{i} N_{i} N_{i}^{T}+\sqrt{2 \alpha_{i}} X & * \\
\left(N_{C_{i}} X+N_{D_{i}} Y\right)^{T} & -\delta_{i} I
\end{array}\right)<0,
$$

where $\alpha=\sum_{i=1}^{m} \alpha_{i}$,

$\Pi_{11}=(A X+B Y)^{T}+(A X+B Y)+\gamma N_{0} N_{0}^{T}$,

$\Pi_{31}=\left[\left(C_{1} X+D_{1} Y\right)^{T},\left(C_{2} X+D_{2} Y\right)^{T}, \cdots,\left(C_{m} X+\right.\right.$ $\left.\left.D_{m} Y\right)^{T}\right]^{T}$, 
$\Pi_{33}=\operatorname{diag}\left(\varepsilon_{1} N_{1} N_{1}^{T}-X, \varepsilon_{2} N_{2} N_{2}^{T}-X, \cdots, \varepsilon_{m} N_{m} N_{m}^{T}-\right.$

$X)$,

$\Pi_{41}=\left[\left(N_{C_{1}} X+N_{D_{1}} Y\right)^{T},\left(N_{C_{2}} X+N_{D_{2}} Y\right)^{T}, \cdots,\left(N_{C_{m}} X+\right.\right.$

$\left.\left.N_{D_{m}} Y\right)^{T}\right]^{T}$,

$\Pi_{44}=\operatorname{diag}\left(-\varepsilon_{1} I,-\varepsilon_{2} I, \cdots,-\varepsilon_{m} I\right)$,

$\Omega_{i}=\left(C_{i} X+D_{i} Y\right)^{T}+C_{i} X+D_{i} Y$.

Proof. The proof is similar to that of Theorem 2 so we only give an outlined one. Set $P=X^{-1}$ and let $V(x)=$ $x^{T} P x$ which obeys (9a). It is easy to show that

$$
\begin{aligned}
\mathcal{L} V(x) & =x^{T}\left[\left(A+B K+N_{0} F_{0}\left(N_{A}+N_{B} K\right)\right)^{T} P\right. \\
& \left.+P\left(A+B K+N_{0} F_{0}\left(N_{A}+N_{B} K\right)\right)\right] x \\
& +x^{T}\left[\sum _ { i = 1 } ^ { m } \left(C_{i}+D_{i} K\right.\right. \\
& \left.+N_{i} F_{i}\left(N_{C_{i}}+N_{D_{i}} K\right)\right)^{T} P\left(C_{i}+D_{i} K\right. \\
& \left.\left.+N_{i} F_{i}\left(N_{C_{i}}+N_{D_{i}} K\right)\right)\right] x .
\end{aligned}
$$
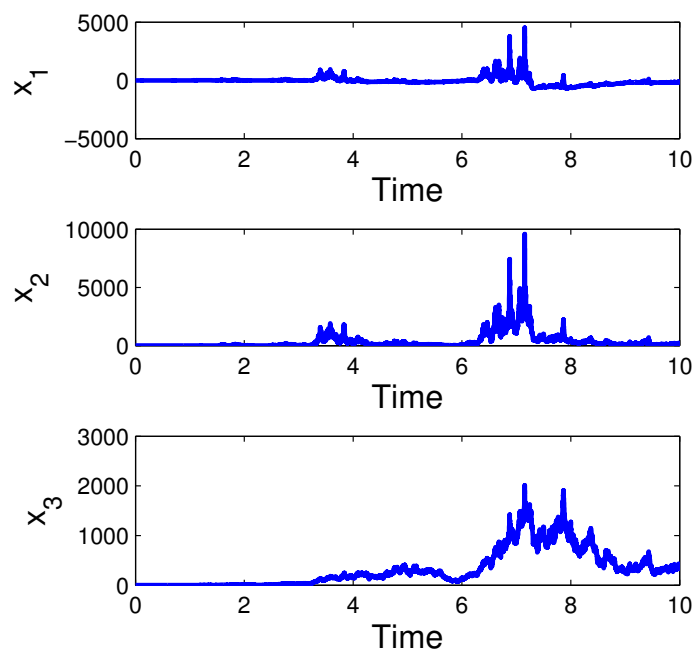

By the fundamental matrix inequalities (see e.g. Wang, Qiao \& Burnham (2002); Xu \& Chen (2002)), we can get

$$
\begin{aligned}
\mathcal{L} V(x) & \leq x^{T}\left[(A+B K)^{T} P+P(A+B K)\right)+\gamma P N_{0} N_{0}^{T} P \\
& \left.+\left(N_{A}+N_{B} K\right)^{T}\left(N_{A}+N_{B} K\right) / \gamma\right] x \\
& +x^{T} \sum_{i=1}^{m}\left[\left(C_{i}+D_{i} K\right)^{T}\left(X-\varepsilon_{i} N_{i} N_{i}^{T}\right)^{-1}\left(C_{i}+D_{i} K\right)\right. \\
& \left.+\left(N_{C_{i}}+N_{D_{i}} K\right)^{T}\left(N_{C_{i}}+N_{D_{i}} K\right) / \varepsilon_{i}\right] x
\end{aligned}
$$

From this we can show in the same way as in the proof of Theorem 2 that LMI (22a) implies inequality (9b) with $c_{2}<\alpha$. Moreover, we have

$$
\begin{aligned}
(\mathcal{H} V(x))^{2} & =\sum_{i=1}^{m}\left(x ^ { T } \left[\left(C_{i}+D_{i} K\right)^{T} P+P\left(C_{i}+D_{i} K\right)\right.\right. \\
& +\left(N_{i} F_{i}\left(N_{C_{i}}+N_{D_{i}} K\right)\right)^{T} P \\
& \left.\left.+P\left(N_{i} F_{i}\left(N_{C_{i}}+N_{D_{i}} K\right)\right)\right] x\right)^{2} .
\end{aligned}
$$

For each $i=1,2, \cdots, m$, it is easy to show

$$
\begin{aligned}
& -\delta_{i} P N_{i} N_{i}^{T} P-\left(N_{C_{i}}+N_{D_{i}} K\right)^{T}\left(N_{C_{i}}+N_{D_{i}} K\right) / \delta_{i} \\
\leq & \left.N_{i} F_{i}\left(N_{C_{i}}+N_{D_{i}} K\right)\right)^{T} P+P\left(N_{i} F_{i}\left(N_{C_{i}}+N_{D_{i}} K\right)\right. \\
\leq & \delta_{i} P N_{i} N_{i}^{T} P+\left(N_{C_{i}}+N_{D_{i}} K\right)^{T}\left(N_{C_{i}}+N_{D_{i}} K\right) / \delta_{i} .
\end{aligned}
$$

From these we can show in the same way as in the proof of Theorem 2 that $(9 \mathrm{c})$ with $c_{3}=2 \alpha$ is guaranteed by $(22 \mathrm{~b})$ or $(22 \mathrm{c})$. Hence the assertion of this theorem follows from Lemma 1.

Example 2. Consider system (20) with $m=1$ and $A=\left(\begin{array}{ccc}-1 & 2 & 0 \\ 1 & 3 & 1 \\ 0 & 0 & 1\end{array}\right), B=\left(\begin{array}{l}1 \\ 1 \\ 0\end{array}\right), C=\left(\begin{array}{ccc}-2 & -1 & -1 \\ -1 & -3 & -0.2 \\ 0 & 0 & -1\end{array}\right)$,

$$
\begin{aligned}
& D=\left(\begin{array}{c}
1 \\
1 \\
-2
\end{array}\right), N_{0}=N_{1}=\left(\begin{array}{ccc}
-0.1 & 0 & 0.1 \\
0.1 & -0.04 & 0 \\
0 & -0.1 & 0
\end{array}\right) \text {, } \\
& N_{A}=\left(\begin{array}{ccc}
0.1 & 0 & -0.1 \\
-0.2 & 0.1 & 0 \\
0 & 0.1 & -0.1
\end{array}\right), N_{B}=\left(\begin{array}{c}
-0.2 \\
0.1 \\
0
\end{array}\right), N_{C_{1}}= \\
& \left(\begin{array}{ccc}
-0.1 & 0 & 0.2 \\
0.1 & -0.2 & 0 \\
0.1 & -0.3 & 0.2
\end{array}\right), N_{D_{1}}=\left(\begin{array}{c}
-0.1 \\
0.2 \\
0.1
\end{array}\right) \text {. It is clearly that }
\end{aligned}
$$

this system cannot be stabilised in mean-square sense. However, setting $h=1$, and for $\alpha=\alpha_{1}=h, 2 h, \cdots$, solving LMIs (22a) and (22b) we find the feasible solution for the a.s. stability:

$$
\alpha=20, X=\left(\begin{array}{ccc}
1.4076 & 2.6818 & -0.7770 \\
2.6818 & 5.6305 & -2.0732 \\
-0.7770 & -2.0732 & 6.6563
\end{array}\right) \text {, }
$$

$K=(-4.63862 .73831 .5736), \gamma=5.5793, \varepsilon_{1}=0.8032, \delta_{1}=$ 3.4642. The corresponding simulation results shown in Fig. 3 and Fig. 4 support the stabilisation result.

\section{Conclusion}

In this paper we have developed a class of LMI conditions for designing a feedback controller to stabilize a given unstable stochastic system in the sense of almost sure exponential stability. These LMI conditions are easy to be checked in practice by using MATLAB Toolbox. Moreover, the control structure in this paper appears not only in drift part but also in diffusion part of the underlying stochastic system. 

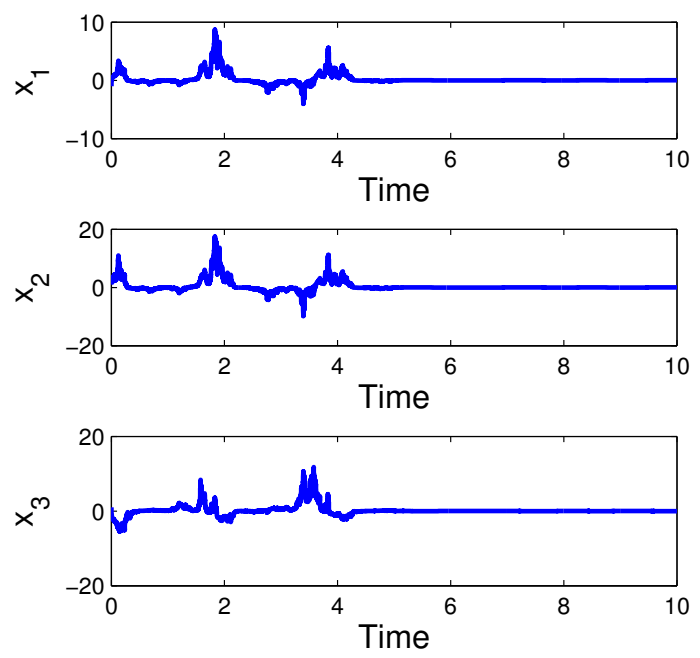

Fig. 4. Robust a.s. Stabilization

\section{Acknowledgements}

The authors would like to thank the referee and the associated editor for their helpful comments and suggestions. This work is supported by Royal Society / KC Wong Fellowship (UK) under Grant RD3485.

\section{References}

Arnold,L., Crauel, H., \& Wihstutz, V. (1983). Stabilization of linear systems by noise. SIAM J. Control. Optim, 21(3), 451-461.

Appleby, J.A.D., \& Mao, X. (2005). Stochastic stabilisation of functional differential equations. Systems $\mathbb{E}$ Control Letters, 54(11), 1069-1081.

Boyd,S., El Ghaoui,L.,Feron E., \& Balakrishnan, V.(1994). Linear Matrix Inequalities in Systems and Control Theory, Philadelphia, PA, USA: SIAM.

Caraballo, T. , \& Robinson, J.C. (2004). Stabilisation of linear PDEs by Stratonovich noise. Systems $\& 3$ Control Letters, 53(1), 41-50.

Chen, B.-S. \& Zhang, W. (2004). Stochastic $H_{2} / H_{\infty}$ control with state-dependent noise. IEEE Transactions on Automatic Control, 49(1), 45 - 57.

Deng, H., \& Krstic, M.(1997). Stochastic nonlinear stabilization-Part I: A backstepping design.Syst. Control Lett., 32, 143-150.

Dragan,V., Morozan,T., \& Stoica, A. (2004). $H^{2}$ Optimal control for linear stochastic systems. Automatica, 40, 1103-1113.

El Ghaoui, L. (1995). State-feedback control of systems with multiplicative noise via linear matrix inequalities. Systems \& Control Letters, 24(3), 223-228.

Ezzine,J.,\& Kavranoglyu,D.(1997). On almost-sure stabilization of discrete-time jump parameter systems: an LMI approach. Int. J. Control, 68(5),1129-1146.

Fang, Y.(1997). A new general sufficient condition for almost sure stability of jump linear systems. IEEE Trans. Automatic Control, 42(3), 378-382.

Fang, Y., \& Loparo,K. A. (2002).Stabilization of continuous-time jump linear systems.IEEE Trans. Automatic Control, 47(10), 1590-1603.

Fernholz, R., \& Karatzas, I. (2005). Relative arbitrage in volatility-stabilized markets, Annals of Finance, 1(2), $149-177$.

Hinrichsen, D., \& Pritchard,A.J. (1998). Stochastic $H^{\infty}$. SIAM J. Control Optim., 36, 1504-1538.

Khasminski, R. Z.(1980). Stochastic stability of Differential Equations, Rockville, Maryland: S\&N International Publisher.

Kwiecińska, A. (2002). Stabilization of evolution equations by noise, Proceedings of Amarican Mathematical Society, 130(10), 3067-3074.

Lee,J.W., \& Dullerudb,G. E.(2006). Uniform stabilization of discrete-time switched and markovian jump linear systems. Automatica, 42, $205-218$.

Mao X.(1997). Stochastic Differential Equations and Applications, Chichester, England, UK: Horwood Publishing.

Mao, X.(1994). Stochastic stabilization and destabilization. Syst. Control Lett., 23,279-290.

Nolan, V. J., \& Sri Namachchivaya, N.(1999). On almost-sure stability of linear gyroscopic systems. Journal of sound and vibration, 227(1), 105-130.

Pan, Z., \& Basar ,T. (1999). Backstepping controller design for nonlinear stochastic systems under a risksensitive cost. SIAM J. Control Optim., 37 (3), 957995.

Pardoux, E., \& Wihstutz,V. (1992). Lyapounov exponent of linear stochastic systems with large diffusion term. Stochastic Processes and their Applications, 40, 289-308.

Roberts, J. B., \& Spanos, P. D. (1986). Stochastic averaging: An approximate method of solving random vibration problems. Internat. J. Non-linear Mesh, 21, 111-134.

Tylikowski, A.(2005). Stabilization of beam parametric vibrations with shear deformations and rotary inertia effects international. Journal of solids and structures, 42, 5920-5930.

Wang,Z., Qiao,H., \& Burnham,K.J.(2002). On stabilization of bilinear uncertain time-delay stochastic systems with Markovian jumping parameters. IEEE Trans. Automat. Contr., 47, 640 - 646.

$\mathrm{Xu}, \mathrm{S} ., \&$ Chen,T.(2002). Robust $H_{\infty}$ control for uncertain stochastic systems with state delay. IEEE Trans. Automat. Contr., 47, 2089-2094.

Xu,S., Shi,P., Chu, M.,\& Zou,Y.(2006). Robust stochastic stabilization and $H_{\infty}$ control of uncertain neutral stochastic time-delay systems. Journal of Mathematical Analysis and Applications, 314(1), 1-16.

Yuan,C., \& Mao, X.(2004). Robust stability and controllability of stochastic differential delay equations with Markovian switching. Automatica, 40, 343-354.

Yuan,C., \& Lygeros,J.(2005) On the exponential stability of swithing diffusion processes. IEEE Trans. Au- 
tomatic Control, 50(9),1422-1426.

Yue, D., \& Han, Q.-L.(2005). Delay-dependent exponential stability of stochastic systems with time-varying delay, nonlinearity, and Markovian switching. IEEE Transactions on Automatic Control, 50(2), 217 - 222. 